\title{
Remembering Prof. Hassan Saidi: The Pillar for Young Anatomists and Trainee Doctors
}

\author{
Brian Ngure Kariuki, Beda Olabu, Isaac Cheruiyot, Jeremiah Munguti, Moses Obimbo, Julius Ogeng'o \\ School of Medicine, University of Nairobi
}

Correspondence to: Dr. Brian Ngure Kariuki, PO Box 3019700100 Nairobi; email: kariukib88@gmail.com

Key words: Anatomy training, Intercalated degree, Mentor

Ann Afr Surg. 2019; 16(1):38-39

DOI: http://dx.doi.org/10.4314/aas.v16i1.9

The late Prof. Hassan Saidi (rest in peace) was a father, husband, an administrator and a surgeon. To many in the medical fraternity, he was a teacher and a mentor. As a mentor, many know of the impact he had on resident surgeons in training, but his contribution to shaping the minds of young anatomists and trainee doctors is often underestimated (1). Prof. Saidi taught in the Department of Human Anatomy, University of Nairobi, for 28 years ( 7 of which he was the chairman). He had also served previously as the coordinator for the Bachelor of Medicine and Surgery (MBChB) and Intercalated Bachelor of Science, Human Anatomy programs (BSc Anat) (2).

He continued to keep close contact with the progress of these programs despite completing his term as coordinator. $\mathrm{He}$ would go on to leave a lasting impression on his students. For example, Prof. Saidi left an indelible mark among his students pursuing BSc (Anatomy) program, an intense oneyear research-based program offered to top undergraduate students at the Department of Human Anatomy (3). The students would normally take a detour from their medical training to focus on anatomy. Prof. Saidi made students of this program not just his mentees but his friends. He made their day-to-day learning activities a priority for him. This included teaching, developing concepts for research, chairing scientific journal club sessions and the practicum in research, which included dissection and histology techniques.

Professor employed a problem-based approach to learning: he presented his students with a unique set of problems. Often his questions seemed simple but they required integration of knowledge across different disciplines. For example, he would ask: "What are the dimensions of the longest axon in the body?" during the first week of dissecting the brain and spinal cord. Such a question needs in-depth understanding of tractology and the length of the spinal cord relative to the subject's height. His ability to critically appraise students' research proposals was also impressive. He was always
Funding: None

(C) 2019 Author. This work is licensed under the Creative Commons Attribution 4.0 International License.

available for consultation and made time to go through all proposal drafts. Most of his mentees were accustomed to his "so what?" questions, which were meant to stimulate critical thinking on the justification and or the significance of their work.

Professor remained visionary as a leader and mentor (4). He always sought remedies to problems and would play a key role in solving them. Doctors in their residency would later go on to have an easy time developing their research topics after the compulsory Friday morning journal club sessions that he chaired. For the BSc (Anatomy) program, he championed the transition of the dark room for photography to a digital one. This allowed students to navigate the histological part of their projects with a lot more ease while producing quality results. Noting the importance of a positive attitude in academia, Professor always had wise counsel. He would go on to say, "If it is lack of knowledge, that we will train you to have, but if it is attitude, then it will be hard for us to intervene." He was committed to producing the best: a cohort of clinician-scientists.

"In this training, we bring you in, we break you, and repackage you to be a scientist," he often said.

Unique to a fault, Prof. Saidi was a compassionate mentor who took time to know his mentees intimately. In addition to providing academic support, he sought to know their social and economic situations, going the extra mile to step in when his student was financially struggling. His humble nature would see him take out his students for a meal, to watch a football match, attend a wedding or even a graduation party. Many students have learned and benefited from his unorthodox style of mentorship and from it a unique way of thinking has been cultured. The challenge now rests on his mentees - the students who stood on the shoulders of this great giant - to make a difference and ensure his legacy in teaching and mentorship lives on. 


\section{References}

1. Kobe IO. Finding the optimal paradigm in surgical training and mentorship: Prof. Hassan's example. Ann Afr Surg. 2018; 15(2):46-7.

2. Green A. Saidi Hassan. The Lancet. 2017; 390(10102):1580

3. University of Nairobi, Department of Human Anatomy, Bachelor of Science Human Anatomy course description. http://humananatomy.uonbi.ac.ke//uon degrees details/684\#. reg_anchor 684 908

4. Ojuka DK. Contribution of Prof Saidi to surgical education in Kenya. Ann Afr Surg. 2018; 15(2):70-2.

\section{Conflicts of Interest}

Dr. Kariuki served as an intern at the journal. He was excluded from the meetings that discussed the paper. 